cells. In PDT, a light-sensitive molecule is delivered to a target cell and activated with light of a specific wavelength. This causes cell death through the production of reactive oxygen species.

Methods The anti-FAP antibody $28 \mathrm{H} 1$ was conjugated with the photosensitizer IRDye700DX (28H1-700DX). In vitro PDT assays were performed with 3 T3 fibroblasts stably transfected with FAP. 3T3-FAP cells were incubated with 28H1$700 \mathrm{DX}$ or a control conjugate for 4 hours, and exposed to varying $690 \mathrm{~nm}$ light doses. Subsequently, cell viability was measured using the CellTiter-Glo assay. For in vivo biodistribution, 5 days after onset of antigen-induced arthritis (AIA) C57Bl6 mice were injected with $28 \mathrm{H} 1 \pm 700 \mathrm{DX}$ labelled with radioactive Indium for quantification purposes (expressed as $\%$ injected dose per gram tissue $(\% \mathrm{ID} / \mathrm{g}))$. For the PDT treatment experiment, arthritic mice were injected at day 5 of AIA with 28H1-700DX or PBS and exposed to 50 or $90 \mathrm{~J} / \mathrm{cm}^{2}$ light 24 hours post injection. Joints were isolated at day 10 for histological analysis.

Results To assess PDT efficacy, we applied $13.7 \mathrm{~J} / \mathrm{cm} 2$ light exposure to 3T3-FAP cells incubated with $6.67 \mathrm{pM} \mathrm{28H1-}$ 700DX, which significantly reduced cell viability $(89.27 \%$ \pm 2.48 compared to control $(\mathrm{p}<0.001))$. No cell death was observed with the control 700DX-conjugate.

Conjugating the anti-FAP antibody to 700DX changed the in vivo biodistribution of the antibody, with a higher accumulation in the liver $(27,06 \pm 0,95 \% \mathrm{ID} / \mathrm{g}$ vs. $6,08 \pm 0,42 \% \mathrm{ID} / \mathrm{g}$ with control $(\mathrm{p}<0,001))$ and lower blood levels $(5,32 \pm 0,36 \%$ $\mathrm{ID} / \mathrm{g}$ vs. $12,72 \pm 0,80 \% \mathrm{ID} / \mathrm{g}$ with control $(\mathrm{p}<0,001))$. Accumulation in the arthritic joints was not significantly different. Histological analysis of the PDT-treated mouse knee joints is ongoing.

Conclusions We have demonstrated fibroblast-specific cell death using 700DX-conjugated $28 \mathrm{H} 1$ PDT, indicating FAPbased PDT as a promising new tool in treating RA. Furthermore, we demonstrated that adding 700DX results in faster liver clearance of the antibody, but does not affect uptake in the inflamed knee joint. Future research will further elucidate the applicability of our conjugate for PDT in animal models of RA.

Disclosure of interest None declared

\section{P108 EXPLORING THE MOLECULAR BASIS OF GENDER BIAS IN SYSTEMIC LUPUS ERYTHEMATOSUS (SLE)}

\footnotetext{
${ }^{1,2} \mathrm{D}$ Kosmara, ${ }^{3} \mathrm{~N}$ Panousis, ${ }^{4} \mathrm{~A}$ Banos, ${ }^{1,2} 2 \mathrm{p}$ Sidiropoulos, ${ }^{3,4} \mathrm{E}^{4}$ Dermitzakis, ${ }^{4} \mathrm{D}$ Boumpas, $1,2 \mathrm{G}$ Bertsias. "Laboratory of Rheumatology, University of Crete Medical School, Greece; IInstitute of Molecular Biology-Biotechnology, FORTH, Iraklio, Greece; ${ }^{3}$ Department of Genetic Medicine and Development, University of Geneva Medical School, Geneva, Switzerland; ${ }^{4}$ Biomedical Research Foundation of the Academy of Athens, Athens, Greece
}

\subsection{6/annrheumdis-2018-EWRR2018.123}

Background/objectives SLE features a substantially greater frequency in females than in males (ratio ranging 7:1-15:1). By contrast, males tend to suffer from more severe disease. ${ }^{1-3}$ Understanding the molecular basis of SLE variability and sexual dimorphism may advance our understanding of disease pathogenesis and assist the development of personalized treatments. We performed full transcriptome analysis (RNA-seq) to monitor for differentially expressed genes (DEGs) between male and female SLE patients that are not differentially expressed between male and female healthy subjects, thus identifying a gender-biased molecular signature specific for the disease.

Materials and methods Whole blood RNA was extracted from SLE patients and healthy individuals. Paired-end mRNA sequencing was performed using the Illumina HiSeq2000 platform. A list of DEGs in SLE males versus females were generated using 5\% false discovery rate (FDR). To increase the specificity of our results, we generated a similar list of DEGs in healthy males versus females using a 50\% and 90\% FDR cut-off, and the two lists were intersected.

Results We studied 142 SLE patients with diverse levels of disease activity/severity (120 SLE females, 22 SLE males) compared to 58 matched healthy volunteers (48 healthy females, 10 healthy males). We identified 39 genes which were significantly differentially expressed in SLE males versus females. Notably, 6 of these genes were not differentially expressed in healthy males versus females at either 50 or $90 \%$ FDR, highlighting a potential role in disease sexual dimorphism. The proteins encoded by these genes are implicated in various biological processes such as transcriptional regulation and DNA damage repair (SMC1A), lipoprotein particles catabolism (APOE), glutathione biosynthesis and metabolism (OPLAH), correct composition of bone and cartilage matrix (ARSD), whereas 2 of these genes do not code for proteins (MTCO2 and FRG1B). Further validation of these genes is in progress. Conclusions A gender-biased molecular signature specifically associated with SLE was unraveled. Further investigation of the molecular pathways which are associated with these genes will give us insights for the molecular basis of gender bias in SLE and lead to novel, more effective treatments tailored for male and female patients.

\section{REFERENCES}

1. Margery-Muir AA, et al. Gender balance in patients with systemic lupus erythematosus. Autoimmun Rev 2017:16(3):258-268.

2. Boodhoo KD, Liu S, Zuo X. Impact of sex disparities on the clinical manifestations in patients with systemic lupus erythematosus: A systematic review and metaanalysis. Medicine (Baltimore) 2016;95(29):e4272.

3. Gergianaki I, et al. Epidemiology and burden of systemic lupus erythematosus in a Southern European population: Data from the community-based lupus registry of Crete, Greece. Ann Rheum Dis 2017.

\section{P109 CLINICAL DEVELOPMENT OF A NOVEL STRATEGY TO MITIGATE BIOLOGIC IMMUNOGENICITY: MONTHLY DOSING OF A PEGYLATED URICASE WITH SVP-R ENABLES SUSTAINED REDUCTION OF SERUM URIC ACID (SUA) LEVELS BY MITIGATING FORMATION OF ANTI- DRUG ANTIBODIES (ADAS)}

${ }^{1} \mathrm{E}$ Sands*, ${ }^{2} \mathrm{~A}$ Kivitz, ${ }^{1} \mathrm{~W}$ DeHaan, ${ }^{1} \mathrm{~L}$ Johnston, ${ }^{1} \mathrm{TK}$ Kishimoto. ${ }^{1}$ Selecta Biosciences, Watertown; ${ }^{2}$ Altoona Centre for Clinical Research, Altoona, USA

\subsection{6/annrheumdis-2018-EWRR2018.124}

Introduction Pegylated uricases are a promising but highly immunogenic therapy for severe gout. Preclinical studies have shown the ability of synthetic vaccine particles containing rapamycin (SVP-R) to inhibit the formation of ADAs against pegsiticase, a pegylated uricase. ${ }^{1}$ Here we report initial data on the safety, immunogenicity and activity of an ongoing Phase 2 study of SEL-212, a novel combination therapy consisting of pegsiticase and SVP-R.

Objectives Evaluate the ability of monthly doses of SEL-212 to mitigate the immunogenicity of pegsiticase and enable sustained control of sUA in gout patients. 
Methods Patients with symptomatic gout and elevated sUA $(\geq 6 \mathrm{mg} / \mathrm{dL})$ were treated with fixed doses of pegsiticase $(0.2 \mathrm{mg} / \mathrm{kg}$ or $0.4 \mathrm{mg} / \mathrm{kg})$ alone or co-administered with SVP-R $(0.05,0.08$, or $0.1 \mathrm{mg} / \mathrm{kg})$. SEL-212 was infused in 28 day cycles $\mathrm{x} 3$ doses followed by challenge with pegsiticase alone on 28 day cycles $\mathrm{x} 2$ doses. Safety, tolerability, sUA, and ADAs were monitored

Results In the SEL-212 Phase 1b study, 70\% of patients administered $0.4 \mathrm{mg} / \mathrm{kg}$ pegsiticase with a mid-dose of $0.1 \mathrm{mg} /$ kg SVP-R showed low or no ADA formation correlating with sustained low sUA levels for at least 30 days after a single dose, compared to $20 \%$ for patients treated with pegsiticase alone. In the ongoing Phase 2 study, the majority of patients receiving $0.1 \mathrm{mg} / \mathrm{kg}$ SVP-R administered with either 0.2 or $0.4 \mathrm{mg} / \mathrm{kg}$ pegsiticase also showed low or no ADAs and maintained low sUA levels after 3 monthly doses of SEL-212, indicating sustained activity with repeated doses of SEL-212. However after 2 subsequent doses of pegsiticase alone, a drop in activity was noted. These data suggest that either a higher dose of SVP-R or the addition of SVP-R at the 4th and 5th dose may be required to sustain activity through 5 months. Currently patients are being dosed with $0.15 \mathrm{mg} / \mathrm{kg}$ SVP-R, a dose level which enabled sustained control of sUA levels in all patients in Phase 1b. SEL-212 was generally well tolerated and associated with a low rate of gout flare rates compared to those treated with pegsiticase alone.

Conclusions SVP-R showed a dose-dependent reduction in ADAs and enabled sustained control of sUA with repeated dosing of SEL212. SVP-R is a promising approach to prevent the formation of ADAs against immunogenic biologic therapies.

\section{REFERENCE}

1. Kishimoto TK, et al. Nature Nanotechnol 2016;11:890-899.

Acknowledgements We thank the patients that participated in these studies, the clinical study site investigators, and the entire SEL-212 project team.

Disclosure of interest E. Sands Employee of: Selecta Biosciences, A. Kivitz: None declared, W. DeHaan Employee of: Selecta Biosciences, L. Johnston Employee of: Selecta Biosciences, T. Kishimoto Employee of: Selecta Biosciences

\section{P110 TOFACITINIB IS ASSOCIATED WITH AN IMPAIRED FUNCTION OF NK CELLS AND A DEFECTIVE IMMUNOSURVEILLANCE AGAINST B-CELL LYMPHOMAS}

${ }^{1} \mathrm{G}$ Nocturne*, ${ }^{2} \mathrm{~J}$ Pascaud, ${ }^{2} \mathrm{~B}$ Ly, ${ }^{2} \mathrm{~F}$ Tahmasebi, ${ }^{2} \mathrm{~S}$ Boudaoud, ${ }^{1} \mathrm{R}$ Seror, ${ }^{3} \mathrm{~L}$ Stimmer, ${ }^{1} \mathrm{X}$ Mariette. ${ }^{1}$ Université Paris Sud; ${ }^{2}$ INSERM, Le Kremlin Bicetre; ${ }^{3} \mathrm{CEA}$, Fontenay aux Roes, France

\subsection{6/annrheumdis-2018-EWRR2018.125}

Introduction Patients with rheumatoid arthritis (RA) are exposed to an increased risk of lymphoma and the impact of treatments is difficult to assess. Tofacitinib, an oral Janus Kinase (Jak) 3 and 1 inhibitor that has shown positive results in RA patients, may impair NK-cell function due to its inhibitory action on IL2 and IL15 signalling.

Objectives Given the fact that NK cells have been recently shown to participate to anti-lymphoma immunosurveillance, we aimed to assess if tofacitinib might impact NK-cell function and anti-lymphoma activity in vitro and in vivo in BAFF transgenic mice (a model of $\mathrm{B}$ cell autoimmunity associated with an increased risk of lymphoma).

Methods We have studied the consequences of in vitro exposure of NK to tofacitinib (10, 50 and $100 \mathrm{nM})$ or to DMSO (vehicle) during 6 days in presence of IL-2 $(200 \mathrm{UI} / \mathrm{ml})$ : phenotype has been studied and then cytotoxicity against 2 nonHodgkin B-cell lymphoma cell lines [Farage $(\mathrm{EBV}+)$ and SUDHL4 (EBV-)] was assessed. In addition, BAFF transgenic mice were treated for 6 months with tofacitinib $(2.25 \mathrm{mg} / \mathrm{kg} / \mathrm{d}$ $\mathrm{n}=11 ; 4.5 \mathrm{mg} / \mathrm{kg} / \mathrm{d} \mathrm{n}=10$ ) or vehicle (PEG: DMSO, $\mathrm{n}=6$ ). Incidence of lymphoma was assessed by histologic examination using a composite score.

Results Firstly, we did not observe difference concerning the survival of NK cells in presence of tofacitinib or vehicle after 6 day culture. Secondly, we observed that culture in presence of tofacitinib was associated with a decreased level of activation with a dose effect. In addition, we observed a decreased expression of activating receptors such as NKp30, NKp44 and NKG2D. Last, we found that tofacitinib blocked NK cell maturation as observed with the significant decreased expression of CD57 on NK cells exposed to tofacitinib at 50 and $100 \mathrm{nM}$. These phenotypic abnormalities were associated with an impaired function of NK as assessed by co-culture: degranulation and cytotoxicity were significantly decreased after exposure to tofacitinib. In BAFF transgenic mice, the crude mortality and incidence of lymphoma did not differ between the 3 groups of treatment.

Conclusions This study demonstrates that tofacitinib treatment negatively impact the state of activation, maturation and functions of NK cells. These defects were not associated with a higher incidence of lymphoma in BAFF $\mathrm{Tg}$ mice after 6 months of exposure. However, this negative impact of tofacitinib on NK cells might participate to the increased risk of herpes zoster infection in patients treated with tofacitinib and suggest to remains cautious about a possible increased risk of lymphoma.

Disclosure of interest None declared

\section{P111 MESENCHYMAL STEM CELL ENCAPSULATION IN ALGINATE MICRO-PARTICLES FOR INTRA-ARTICULAR INJECTION IN OSTEOARTHRITIS}

${ }^{1} \mathrm{~A}$ Smith, ${ }^{2} \mathrm{~A}$ Des Rieux, ${ }^{3} \mathrm{M}$ Marquis, ${ }^{3} \mathrm{D}$ Renard, ${ }^{1} \mathrm{C}$ Vinatier, ${ }^{1,4 \mathrm{~J}}$ Guicheux ${ }^{*},{ }^{1} \mathrm{C}$ Le Visage. 'INSERM UMR 1229 - RMeS, Regenerative Medicine and Skeleton, STEP Team, University of Nantes, UFR Odontology, Nantes, France; ${ }^{2}$ Louvain Drug Research Institute, Advanced Drug Delivery and Biomaterials, Université Catholique de Louvain, Louvain, Belgium; ${ }^{3}$ UR1268 BIA (Biopolymères Interactions Assemblages), INRA; ${ }^{4}$ PHU 4 OTONN, CHU Nantes, Nantes, France

\subsection{6/annrheumdis-2018-EWRR2018.126}

Introduction Owing to their ability to secrete anti-inflammatory and immuno-modulatory factors Mesenchymal Stromal Cells (MSCs) are an attractive tool for the treatment of osteoarthritis. Considering cell death and the risk of cell leakage upon intra-articular injection, MSCs encapsulation therefore could protect cell from death, avoid cell effusion outside the articular space, and supply a suitable $3 \mathrm{D}$ microenvironment supporting the biological activity of MSCs.

Objectives Our objective was to develop a method of MSC encapsulation compatible with their intra-articular injection through a $26 \mathrm{G}$ needle. 